\title{
Magnetoacoustic waves in the narrowband dm-spikes sources
}

\author{
M. Karlický1 ${ }^{1}$ P. Jelínek ${ }^{2}$, and H. Mészárosová ${ }^{1}$ \\ 1 Astronomical Institute of the Academy of Sciences of the Czech Republic, 25165 Ondřejov, Czech Republic \\ e-mail: karlicky@asu.cas.cz \\ ${ }^{2}$ University of South Bohemia, Faculty of Science, 37005 České Budějovice, Czech Republic
}

Received 19 November 2010 / Accepted 22 February 2011

ABSTRACT

\begin{abstract}
Aims. A new type of analysis of the narrowband dm-spikes in solar radio radiation is introduced to look for magnetoacoustic waves in their sources.

Methods. The Fourier and wavelet methods were used. For the first time, the tadpole structures in the wavelet spectra of this radio emission were searched for.

Results. Fifteen groups of the narrowband dm-spikes, observed during solar flares, were selected and analyzed by the Fourier and wavelet analysis methods. We found that the mean Fourier spectra of these spikes in frequency space are the powerlaws with a powerlaw index in the range $-1.2--1.8$. Furthermore, their wavelet spectra based on time series reveal tadpoles at some frequencies, which indicates the presence of magnetoacoustic waves. These waves are interpreted as propagating through a source of the narrowband $\mathrm{dm}$-spikes. It is proposed that the spikes are generated by driven coalescence and fragmentation processes in turbulent reconnection outflow. This interpretation is supported by a simultaneous observation of drifting pulsating structures (DPSs) and spikes. Finally, modeling of the magnetoacoustic waves and tadpoles in the Harris current sheet supports this interpretation.
\end{abstract}

Key words. Sun: corona - Sun: flares - Sun: radio radiation

\section{Introduction}

The radio narrowband decimetric spikes belong to the most interesting radio bursts with exceptionally high brightness temperatures $\left(T_{\mathrm{b}} \approx 10^{15} \mathrm{~K}\right)$ and short durations $(\leq 0.1 \mathrm{~s}$, see the review by Benz 1986). Their observational characteristics have been described in many papers (e.g. Slottje 1981; Karlický 1984; Fu et al. 1985; Stähli \& Magun 1986; Benz et al. 1982; Zlobec \& Karlický 1998; Mészárosová et al. 2003). On the other hand, the theoretical models can be divided into two groups: a) those based on the plasma emission and acceleration processes (Kuijpers et al. 1981; Tajima et al. 1990; Wentzel 1991; Bárta \& Karlický 2001), and b) those based on the electroncyclotron maser (Holman et al. 1980; Melrose \& Dulk 1982; Vlahos \& Sharma 1984; Winglee et al. 1988; Aschwanden 1990; Fleishman \& Yastrebov 1994; Fleishman \& Melnikov 1998). To distinguish between these two types of models, polarization and harmonic structures of the spikes have also been studied (Güdel 1990; Güdel \& Zlobec 1991; Krucker \& Benz 1994).

Searching for a characteristic bandwidth of individual spikes Karlický et al. (1996, 2000) found that the Fourier transform of the dynamic spectra of spikes have a power-law form with power-law indices close to $-5 / 3$. Based on these results, Bárta \& Karlický (2001) propose that the spikes are generated in turbulent reconnection outflows at positions where the upper hybrid frequency is equal to the low harmonics of the electron cyclotron frequency (double resonance). The instability is driven by electron beams accelerated in the reconnection diffusion region. However, new findings of the cascading (fragmented) reconnection (Bárta et al. 2010a,b) lead us to a partial modification of this model, as seen the following.

Recently, Mészárosová et al. (2009a,b) used a new technique based on the wavelet analysis, to detect, for the first time, the tadpoles in the wavelet spectra (interpreted as the magnetoacoustic waves) in some radio sources. The magnetoacoustic waves are trapped in regions with higher density that act as waveguides. If these waves are impulsively triggered at some given location, then at some distance from the initiation site, these propagating waves (owing to their dispersion characteristics) can be detected as the tadpole structure in their wavelet spectra; for details, see e.g. Nakariakov et al. (2004).

Because the magnetoacoustic waves can be easily triggered in the reconnection and because we proposed that the spikes are generated during the reconnection process (Bárta \& Karlický 2001), we decided to search for the magnetoacoustic waves in the spike sources (tadpoles in the wavelet spectra of radio fluxes), thereby supporting our model.

As shown in the following, we found these tadpoles (waves) in all studied groups of spikes. Then we simulated these waves and tadpoles in the model with the Harris current sheet. Finally, the results are discussed and a conclusion drawn.

\section{Observations and data analysis}

We selected $15 \mathrm{dm}$-radio events with the spikes recorded by the Ondřejov radiospectrograph (Jiřička et al. 1993) during the years 1997-2003 in the frequency range $0.8-2.0 \mathrm{MHz}$. The times of their observations, GOES X-ray, and $\mathrm{H} \alpha$ characteristics of associated events are presented in Table 1. A typical example of these spikes, observed at 12:45:10-12:45:53 UT in 2003 August 5, is shown in Fig. 1.

First, we analyzed all of the selected groups of spikes (Table 1) using the same method as described in the paper by Karlický et al. (1996). The radiospectrograms were resampled 
Table 1. Radio events with the narrowband dm-spikes and characteristics of associated flares.

\begin{tabular}{|c|c|c|c|c|c|c|c|c|c|c|}
\hline \multirow[b]{2}{*}{ No. } & \multirow[b]{2}{*}{ Flare } & \multicolumn{2}{|c|}{ Radio } & \multicolumn{4}{|c|}{ GOES } & \multicolumn{3}{|c|}{$\mathrm{H} \alpha$} \\
\hline & & $\begin{array}{l}\text { Start } \\
\text { [UT] }\end{array}$ & $\begin{array}{l}\text { End } \\
\text { [UT] }\end{array}$ & $\begin{array}{l}\text { Start } \\
\text { [UT] }\end{array}$ & $\begin{array}{l}\text { Max } \\
\text { [UT] }\end{array}$ & $\begin{array}{l}\text { End } \\
\text { [UT] }\end{array}$ & $\begin{array}{l}\text { X-ray } \\
\text { Imp. }\end{array}$ & $\begin{array}{c}\text { Optic. } \\
\text { Imp. }\end{array}$ & Position & $\begin{array}{c}\text { NOAA } \\
\text { AR \# }\end{array}$ \\
\hline 1 & 1997 Sep. 12 & $16: 05$ & $16: 07$ & $16: 00$ & $16: 06$ & $16: 17$ & C6.0 & & & \\
\hline 2 & 1997 Nov. 3 & 09:05 & 09:06 & 09:03 & 09:10 & 09:13 & M1.4 & & & \\
\hline 3 & 2000 Feb. 22 & 13:00 & 13:02 & $12: 46$ & $12: 49$ & $13: 21$ & $\mathrm{C} 2.1$ & & & \\
\hline 4 & 2000 Apr. 15 & $13: 40$ & $13: 41$ & $13: 38$ & $13: 43$ & $13: 50$ & C3.0 & SF & S22 E29 & 8955 \\
\hline 5 & 2000 May 15 & $10: 48$ & $10: 50$ & $10: 46$ & $10: 50$ & $10: 56$ & M1.2 & & & \\
\hline 6 & 2000 May 30 & $10: 29$ & $10: 31$ & 09:54 & $10: 18$ & $10: 40$ & C8.6 & & & \\
\hline 7 & $2000 \mathrm{Jul} .8 \mathrm{a}$ & $07: 32$ & $07: 34$ & $07: 29$ & $07: 34$ & $07: 39$ & C6.1 & $1 \mathrm{~N}$ & N17 W07 & 9070 \\
\hline 8 & 2000 Jul. 8b & 07:58 & 08:00 & $07: 29$ & $07: 34$ & $07: 39$ & C6.1 & SF & S22 E15 & 9073 \\
\hline 9 & 2001 Mar. 25 & 11:07 & 11:09 & $10: 33$ & $11: 16$ & $11: 20$ & M2.6 & $1 \mathrm{~N}$ & N21 E59 & 9401 \\
\hline 10 & 2001 Mar. 28 & 12:07 & $12: 26$ & $12: 03$ & $12: 10$ & $12: 17$ & C6.0 & & & \\
\hline 11 & 2002 Dec. $20 \mathrm{a}$ & $10: 47$ & $10: 49$ & $10: 45$ & $10: 49$ & $10: 53$ & C1.2 & & & \\
\hline 12 & 2002 Dec. $20 b$ & $11: 26$ & $11: 28$ & $11: 25$ & $11: 28$ & $11: 30$ & $\mathrm{C} 2.7$ & & & \\
\hline 13 & 2003 Mar. 18 & $12: 06$ & $12: 08$ & $11: 51$ & $12: 08$ & $12: 20$ & X.1.5 & 1B & S15 W46 & 10314 \\
\hline 14 & 2003 Jun. 10 & $08: 34$ & $08: 37$ & 08:34 & 08:37 & $08: 40$ & M2.7 & $1 \mathrm{~N}$ & N12 W44 & 10375 \\
\hline 15 & 2003 Aug. 5 & $12: 45$ & $12: 46$ & $12: 43$ & $12: 49$ & $12: 51$ & M1.7 & SN & S16 E33 & 10424 \\
\hline
\end{tabular}

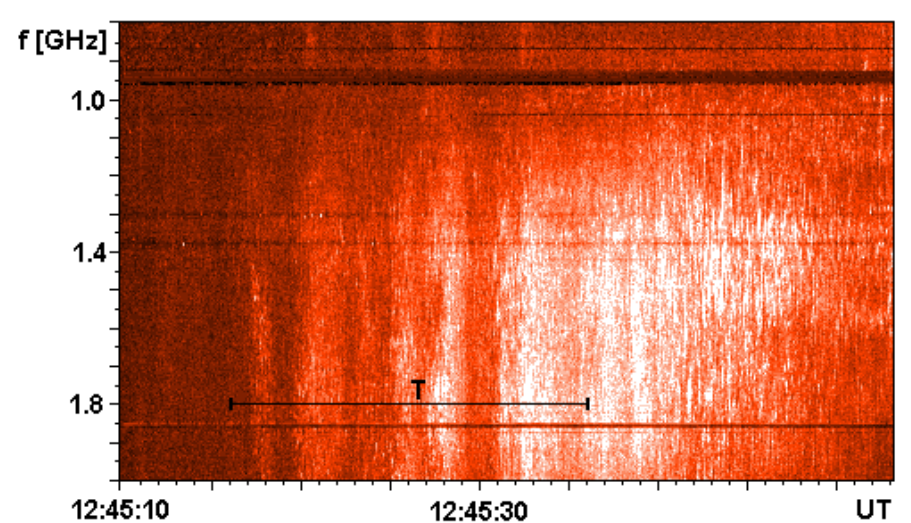

Fig. 1. Typical radio dynamic spectrum $(0.8-2.0 \mathrm{GHz})$ showing the narrowband $\mathrm{dm}$-spikes observed by the Ondrejov radiospectrograph at 12:45:10-12:45:53 UT on 2003 August 5. Tadpole wavelet patterns were recognized in the time interval $T$ (12:45:16-12:45:36 UT).

along the frequency axis according to the formula

$z(f)=H_{\mathrm{n}} \ln \frac{f}{f_{0}}$,

where $z(f)$ is the height in the solar atmosphere in dependence on frequency $f, H_{\mathrm{n}}$ is the density height-scale for the radio emission by the plasma emission mechanism, and $f_{0}=2 \mathrm{GHz}$ in our case (for details see Karlický et al. 1996). After that, the spectrum areas for spikes and background noise were chosen and the time-averaged power spectra along the height axis $P(k)$ (where the wavenumber $k$ is in units of $H_{\mathrm{n}}^{-1}$ ) were computed. The power spectra at high wavenumbers (here for $\log k>2.5$ ) are likely to be affected by the background noise. Therefore the power spectra were "corrected" for the background noise contribution by subtracting this component. This is not a precise method, but it can help us to estimate the behavior of the signal power spectra also at high wave numbers.

The computed power-law index for all spikes groups (second column in Table 2) ranges from -1.2 to -1.8 ( -1.5 on average). An example of the 1-D Fourier spectrum versus the distance scale $k$ (after the substraction of the noise power spectra) for the 2003 August 5 event is presented in Fig. 2. The straight dashed line with the power-law index $-5 / 3$ is added for comparison.

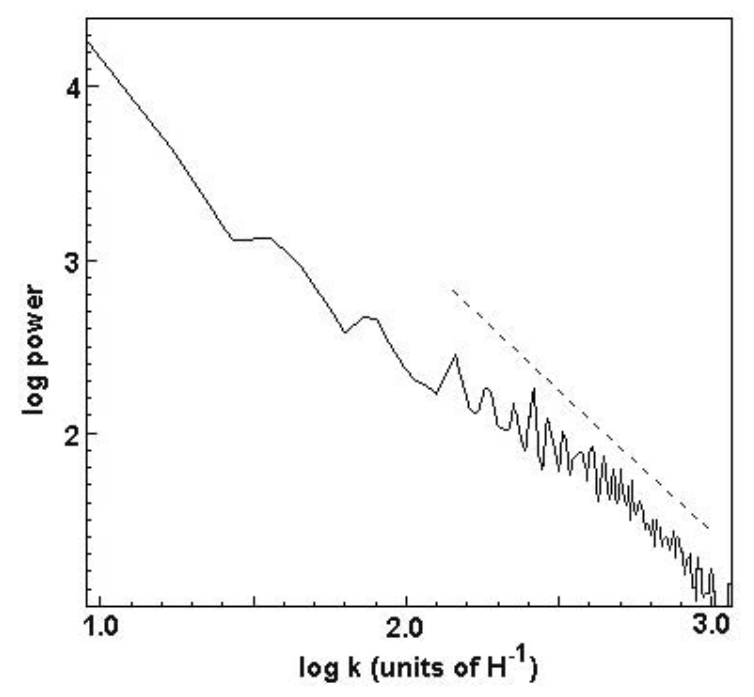

Fig. 2. The 1-D Fourier spectrum for the 2003 August 5 event versus the distance scale $k$ (see text). The straight dashed line denotes the line with the power-law index $-5 / 3$ for comparison.

Table 2. Power-law indices of the Fourier spectra of the narrowband $\mathrm{dm}$-spikes and parameters of associated wavelet tadpoles.

\begin{tabular}{lcccc}
\hline \hline No. & $\begin{array}{c}\text { Spikes } \\
\text { Power-law index } \\
\text { of spikes }\end{array}$ & $\begin{array}{c}\text { Start time } \\
{[\mathrm{UT}]}\end{array}$ & $\begin{array}{c}\text { Tadpoles } \\
\text { Duration } \\
{[\mathrm{s}]}\end{array}$ & $\begin{array}{c}\text { Period } \\
{[\mathrm{s}]}\end{array}$ \\
\hline 1 & -1.8 & $16: 05: 55$ & 20 & 5.1 \\
2 & -1.2 & $09: 07: 10$ & 32 & 5.7 \\
3 & -1.4 & $13: 00: 49$ & 13 & 3.6 \\
4 & -1.7 & $13: 40: 09$ & 27 & 5.8 \\
5 & -1.3 & $10: 48: 33$ & 09 & 3.3 \\
6 & -1.3 & $10: 29: 55$ & 18 & 4.1 \\
7 & -1.3 & $07: 32: 50$ & 23 & 5.0 \\
8 & -1.3 & $07: 58: 51$ & 10 & 3.4 \\
9 & -1.7 & $11: 07: 48$ & 13 & 3.8 \\
10 & -1.5 & $12: 08: 10$ & 16 & 6.0 \\
11 & -1.6 & $10: 47: 59$ & 16 & 3.3 \\
12 & -1.6 & $11: 26: 42$ & 25 & 6.5 \\
13 & -1.8 & $12: 07: 01$ & 23 & 5.5 \\
14 & -1.7 & $08: 35: 51$ & 31 & 4.9 \\
15 & -1.4 & $12: 45: 16$ & 20 & 6.3 \\
\hline mean & -1.5 & & 20 & 4.8 \\
\hline
\end{tabular}



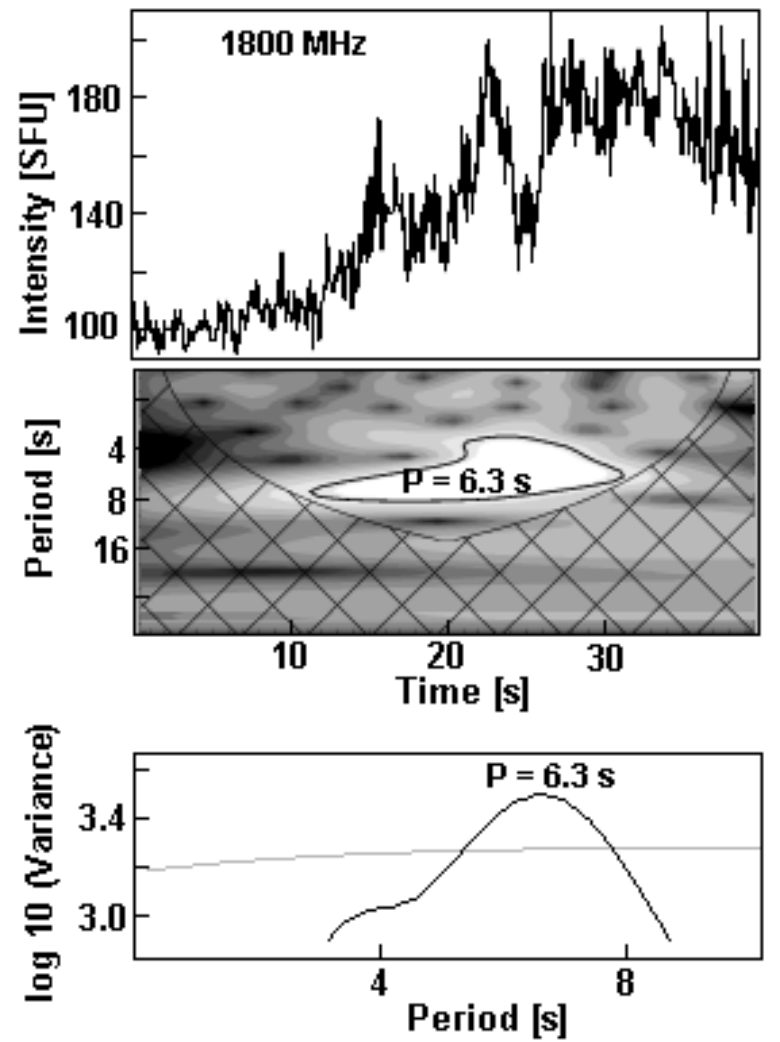

Fig. 3. Typical example of the tadpole detected at the radio frequency $1800 \mathrm{MHz}$ in the interval $T$ (see Fig. 1). Upper panel shows original time series (2003 August 5 event). Middle panel shows its wavelet power spectrum with the tadpole pattern (characteristic period $P=$ $6.3 \mathrm{~s}$ ) outlined by a contour at the $99 \%$ confidence level. Bottom panel shows the global wavelet spectrum (black line) with the maximum for the period $P=6.3$ s. The gray line means the $99 \%$ significance level.

Then, we searched for tadpoles in the wavelet spectra as signatures of magnetoacoustic waves in all spikes events (Table 1) at all recorded frequencies. We used the methods described in the papers by Mészárosová et al. (2009a,b). To be sure that these tadpoles belong to studied spikes we focused only on the dominant tadpoles with the shortest characteristic periods $P<10 \mathrm{~s}$. The basic characteristics of these tadpoles are in Table 2 . The tadpole period $P$ and duration range from 3.4 to $6.5 \mathrm{~s}$ ( $4.8 \mathrm{~s}$ on average) and from 9 to $32 \mathrm{~s}$ (20 s on average), respectively. A typical example is presented in Fig. 3 where the upper panel shows the radio flux observed on $1800 \mathrm{MHz}$ during the 2003 August 5 event in the interval $T$, see Fig. 1. Middle panel shows its wavelet power spectrum with the tadpole pattern (characteristic period $P=6.3 \mathrm{~s}$ ) outlined by contour at the $99 \%$ confidence level. Bottom panel shows the global wavelet spectrum (black line) with the maximum for the period $P=6.3 \mathrm{~s}$. The gray line means the $99 \%$ significance level.

\section{Modeling of magnetoacoustic waves and tadpoles in the narrowband dm-spikes sources}

\subsection{Interpreting the narrowband $d m$-spikes}

As presented above the mean Fourier spectra (made along the frequency) in all studied groups of spikes are the power-law ones with the power-law index in the range $-1.2--1.8$. This (for larger set of spike events than in previous studies) confirms our previous result (Karlický et al. 1996, 2000) that these spectra have a power-law index close to $-5 / 3(=-1.66)$, which in known as the Kolmogorov index of MHD turbulence.

An entirely new result is that in all spike events (at least at limited ranges of frequencies) we found the tadpoles, which indicate the presence of propagating magnetoacoustic waves. Their mean period is about $5 \mathrm{~s}$. Because the magnetoacoustic waves are a natural part of the turbulent outflow, we consider these waves as those supporting the model of spikes in the region of the magnetic reconnection outflows (Bárta \& Karlický 2001).

To interpret these results we propose the model shown in Fig. 4. It is based on "the standard" CSHKP flare model (e.g. Magara et al. 1996 and references therein). In accordance with Shibata and Tanuma (2001), the global current sheet is stretched by rising magnetic rope and fragmented by the tearing-mode instability to smaller and smaller plasmoids (in 2-D O-type magnetic field structures, in 3-D magnetic ropes) and current sheets. This fragmentation (cascading) process produces very narrow current sheets (down to a few meters) with very high current densities, where the anomalous resistivity is generated and fast reconnection occurs. Moreover, in the model, the plasmoids are accumulated and compressed in turbulent reconnection-plasma outflows, especially in the region above the flare loop arcade. These plasmoids have different sizes. They are driven by outflows to interact and coalesce into larger ones and are also fragmented into smaller plasmoids. That is to say, a new current sheet is formed between two merging plasmoids and new smaller plasmoids are generated in this current sheet, on smaller and smaller spatial scales. We called this process driven coalescences and fragmentations (Fig. 4). For more details, see Bárta et al. (2010b).

Furthermore, Karlický \& Bárta (2007) and (Karlický et al. 2010) have shown that each coalescence process accelerates electrons that generate plasma waves, as well as the electromagnetic ones. Considering all these facts, we propose that the narrowband $\mathrm{dm}$-spikes are generated by the driven coalescence and fragmentation processes of plasmoids in the turbulent reconnection outflows. The observation shown in Fig. 5 supports this interpretation. As seen here, the drifting pulsating structures (DPSs) at the $1.0-1.8 \mathrm{GHz}$ frequency range drift towards higher frequencies (towards lower heights in the solar atmosphere), where the spikes are observed. The DPSs indicate the plasmoids moving downwards in the solar atmosphere (Bárta et al. 2008a,b), towards the spikes source, in agreement with the scenario presented in Fig. 4.

Furthermore, we assume that the global reconnection process can be perturbed by e.g. some inhomogeneity in the reconnection inflows and thus to generate magnetoacoustic waves propagating into the spikes source (see Fig. 4). These waves can easily modulate the coalescence processes among plasmoids and thus to modulate electron acceleration and subsequent production of spikes.

To support this interpretation, we take the Harris current sheet as a simple model of the magnetic field and density structure of the global current sheet, which includes reconnection outflows. The current sheets, in which the fast reconnection occurs, are only tiny substructures of the global current sheet, and these substructures are neglected in the following computations. The global current sheet is perturbed at one location, and then at some distant location from the first one, the propagating magnetoacoustic waves and the corresponding tadpoles are analyzed. The parameters of tadpoles depend on the parameters of the model. On the other hand, these simulated tadpoles, which fit the observed ones, give us information about parameters of the global current sheet where the spikes are generated. 


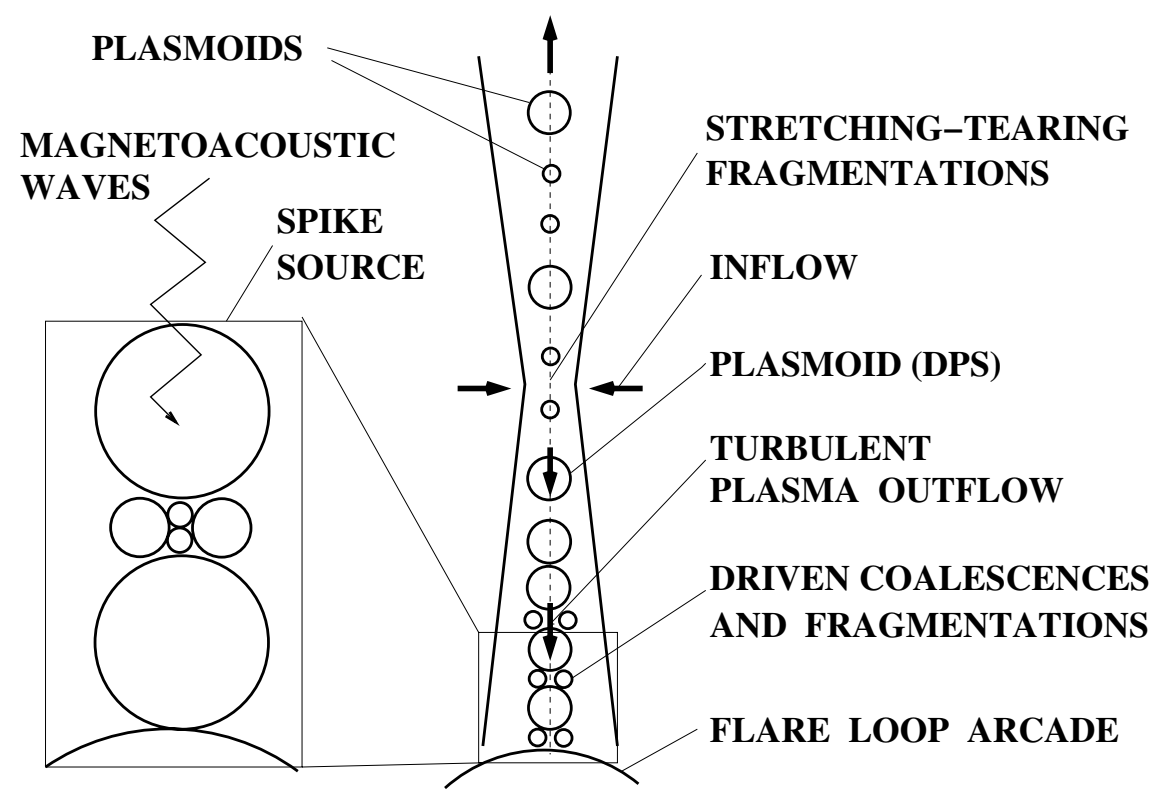

Fig. 4. Scenario of the dm-spike generation in the flare current-sheet reconnection and magnetoacoustic waves propagation. Compare with Fig. 5.

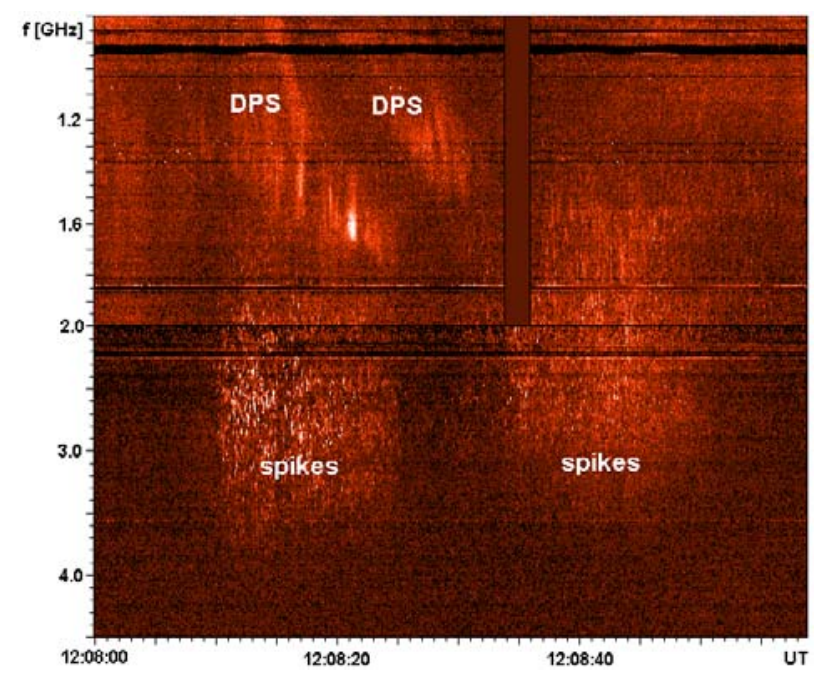

Fig. 5. Composite radio spectrum from two Ondřejov radiospectrographs (0.8-2.0 and 2.0-4.5 GHz) showing the positively drifting pulsating structures (DPSs) and narrowband dm-spikes observed on 2001 March 28 . The $0.8-2.0 \mathrm{GHz}$ spectrum was shortly interrupted at 12:08:34-12:08:36 UT. Compare with Fig. 4.

\subsection{Numerical model}

\subsubsection{Model equations}

In our model we describe plasma dynamics by the ideal magnetohydrodynamic equations (Priest 1982; Chung 2002):

$$
\frac{\partial \varrho}{\partial t}=-\nabla \cdot(\varrho v)
$$

$\varrho \frac{\partial \boldsymbol{v}}{\partial t}+\varrho(\boldsymbol{v} \cdot \nabla) \boldsymbol{v}=-\nabla p+\frac{1}{\mu_{0}}(\nabla \times \boldsymbol{B}) \times \boldsymbol{B}$,

$\frac{\partial \boldsymbol{B}}{\partial t}=\nabla \times(\boldsymbol{v} \times \boldsymbol{B})$,

$\frac{\partial U}{\partial t}=-\nabla \boldsymbol{S}$,

(4) $\Delta t \leq \frac{\operatorname{CFL} \Delta x}{\max \left(c_{\mathrm{s}}+|\boldsymbol{v}|\right)}$,

$\nabla \cdot \boldsymbol{B}=0$.
Here $\varrho$ is a mass density, $\boldsymbol{v}$ flow velocity, $p$ gas pressure, and $\boldsymbol{B}$ is the magnetic field. The plasma energy density $U$ is given by

$U=\frac{p}{\gamma-1}+\frac{\varrho}{2} v^{2}+\frac{B^{2}}{2 \mu_{0}}$

with the adiabatic coefficient $\gamma=5 / 3$, and the flux vector $S$ is expressed as

$\boldsymbol{S}=\left(U+p+\frac{B^{2}}{2 \mu_{0}}\right) \boldsymbol{v}-(\boldsymbol{v} \cdot \boldsymbol{B}) \frac{\boldsymbol{B}}{\mu_{0}}$.

The magnetohydrodynamic Eqs. (2)-(6) were transformed into a flux conserving form, i.e.,

$\frac{\partial \Psi}{\partial t}+\frac{\partial \mathbf{F}(\boldsymbol{\Psi})}{\partial x}+\frac{\partial \mathbf{G}(\boldsymbol{\Psi})}{\partial y}=0$

and were solved numerically. The vector $\boldsymbol{\Psi}$ in our twodimensional case is expressed as

$\boldsymbol{\Psi}=\left(\begin{array}{c}\rho \\ \rho v_{x} \\ \rho v_{y} \\ B_{x} \\ B_{y} \\ U\end{array}\right)$.

The vector functions $\mathbf{F}(\boldsymbol{\Psi})$ and $\mathbf{G}(\boldsymbol{\Psi})$ are too complex to be presented here, but see them at e.g. (Sankaran 2002; Chung 2002).

For the numerical solution of this type of equation, the modified two step Lax-Wendroff algorithm was used. The numerical region was in the $x-y$-plane, implemented at $0 \leq x \leq L$ and $0 \leq y \leq H$ and was covered by a uniform grid with $1250 \times 300$ cells. Open boundary conditions were applied and the time step satisfied the Courant-Friedrichs-Levy condition in the form (Chung 2002):

where $\mathrm{CFL}=0.8$ is called the Courant number. For more details about such simulations, see the papers by Jelínek \& Karlický (2009, 2010). 
M. Karlický et al.: Narrowband dm-spikes

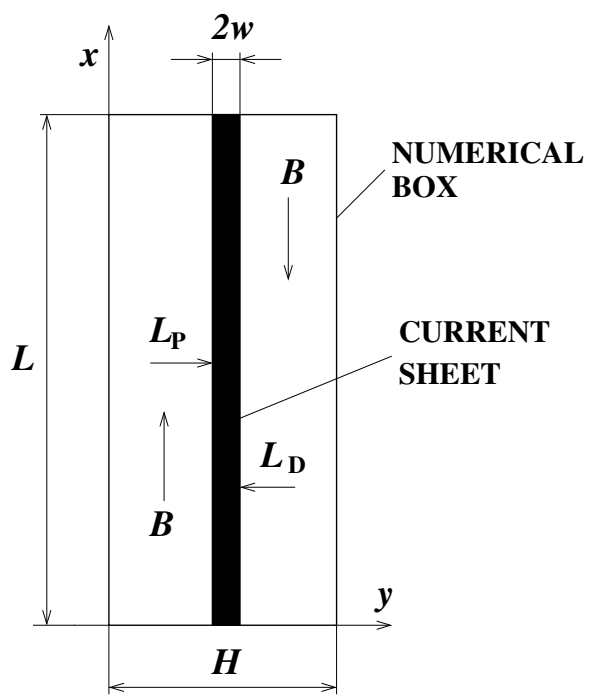

Fig. 6. Sketch of the initial 2-D Harris current sheet considered in simulations. The positions of initial pulse perturbation $\left(L_{\mathrm{P}}\right)$ and data collection $\left(L_{\mathrm{D}}\right)$ are indicated by the arrows. Compare with Fig. 4 .

\subsubsection{Model initial conditions}

Figure 6 schematically shows the initial 2-D current sheet. The magnetic field in such a current sheet is given by the equation:

$\boldsymbol{B}=B_{\text {out }} \tanh \left(\frac{(y-H / 2)}{w}\right) \hat{\boldsymbol{e}}_{\boldsymbol{x}}$,

where $B_{\text {out }}$ is the magnetic field at $y \rightarrow \infty$ and $w$ the half-width of the current sheet. We take the temperature to be the same in the whole numerical box. The plasma density in the current sheet center, where we assume the source of spikes, is determined from the frequency on which the tadpole was found (assuming for simplicity the plasma emission process on the plasma frequency). The other parameters in the current sheet are determined by a choice of the plasma beta parameter $\beta$ in the region far away from the current sheet $(y \rightarrow \infty)$. It follows from the equation

$B_{\text {out }}=\sqrt{\frac{2 \mu_{0} p_{\mathrm{CS}}}{1+\beta}}$,

where $p_{\mathrm{CS}}$ is the kinetic pressure in the center of the current sheet. The initial plasma velocity is assumed to be $\boldsymbol{v}=0$ everywhere in the numerical box.

To generate the magnetoacoustic waves we perturb the current sheet by a pulse in velocities (see Nakariakov et al. 2004):

$v_{y}=A_{0} \frac{y}{\lambda_{x}} \exp \left(-\frac{\left(x-L_{\mathrm{P}}\right)}{\lambda_{x}}\right)^{2} \exp \left(-\frac{(y-H / 2)}{\lambda_{y}}\right)^{2}$,

where $\lambda_{x}$ and $\lambda_{y}$ are the dimensions of the pulse in $x-$ and $y$ - axis, and $A_{0}$ is the amplitude of the initial pulse. The pulse in the velocity was generated at the start of the numerical simulation at $t=0 \mathrm{~s}$. The position of the initial pulse was located at the point $\left(L_{\mathrm{P}}, H / 2\right)$. On the other hand, the propagating magnetoacoustic waves were detected at the point $\left(L_{\mathrm{D}}, H / 2\right)$, see Fig. 6.

\subsection{Results of numerical simulations}

In our simulations we tried to find the best fit of the modeled tadpole with the observed one. Among many possible parameters, we selected some of them as fixed: the temperature is
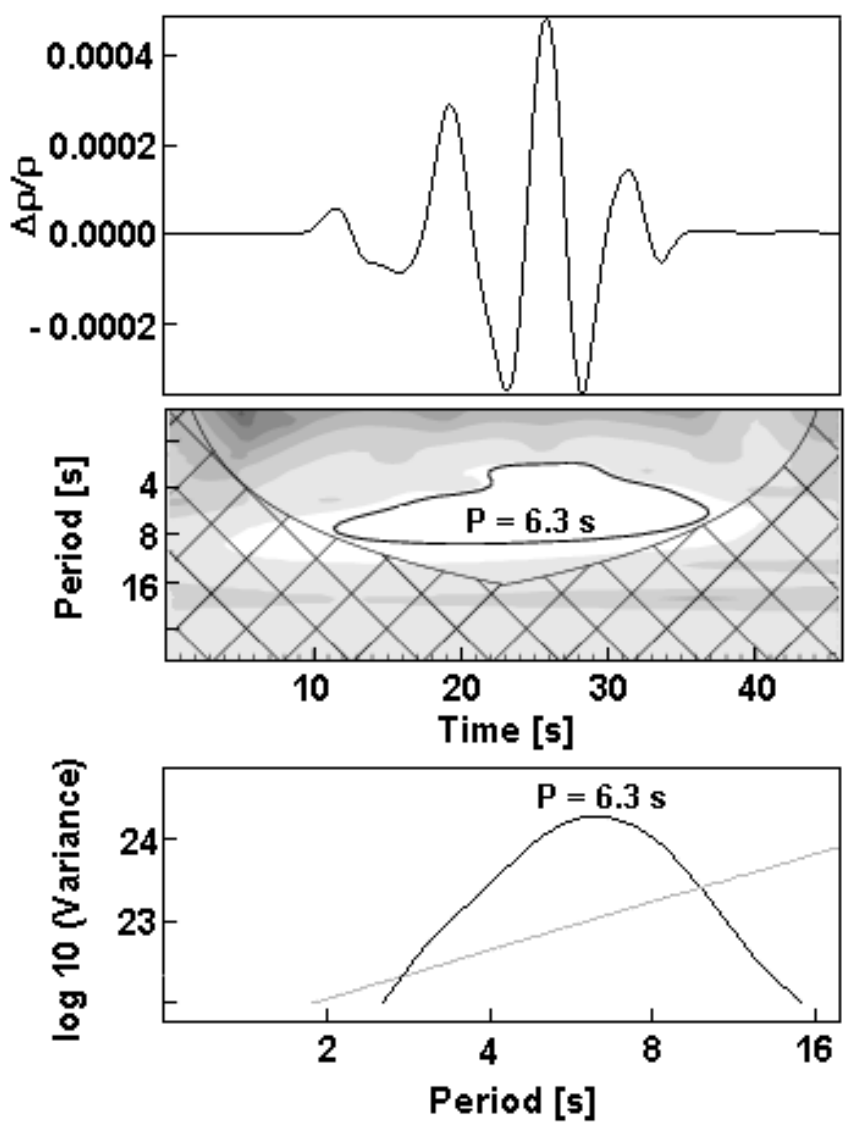

Fig. 7. The modeled tadpole that fits the observed one shown in Fig. 3. Upper panel shows a time evolution of the mass density at the detection point (spikes source), where $L_{\mathrm{P}}-L_{\mathrm{D}}=8.33 \mathrm{Mm}$. Middle panel shows the corresponding wavelet power spectrum with the tadpole pattern (characteristic period $P=6.3 \mathrm{~s}$ ) outlined by contour at the $99 \%$ confidence level. Bottom panel shows the global wavelet spectrum (black line) with the maximum for the period $P=6.3 \mathrm{~s}$. The gray line indicates the $99 \%$ significance level.

$T=1 \times 10^{7} \mathrm{~K}$, the plasma beta parameter outside the current sheet is $\beta=0.1$, the length of the current sheet is $L=100 \mathrm{Mm}$, the plasma density in the current sheet center (at $H / 2)$ is $n_{\mathrm{e}}=$ $4.0 \times 10^{16} \mathrm{~m}^{-3}$, the location of the initial perturbation is $L_{\mathrm{P}}=L / 2$, and the parameters of the perturbation pulse are $\lambda_{x}=1.0 \mathrm{Mm}$, $\lambda_{y}=1.5 \mathrm{Mm}$, and $A_{0}=1.5 \times 10^{4} \mathrm{~m} \mathrm{~s}^{-1}$.

To find the best fit, the halfwidth of the current sheet $w$ and distance between $L_{\mathrm{P}}$ and $L_{\mathrm{D}}$ are varied. We found that (i) increasing the half-width of the current sheet increases the characteristic period of tadpoles; (ii) decreasing the distance between $L_{\mathrm{P}}$ and $L_{\mathrm{D}}$ makes the tadpole shorter. With these facts, the "best" fit was found for $w=1 \mathrm{Mm}$ and the distance $L_{\mathrm{P}}-L_{\mathrm{D}}=8.33 \mathrm{Mm}$. The "best" fitted tadpole is shown in Fig. 7 (compare with that in Fig. 3), where the upper panel shows a time evolution of mass density at the detection point, i.e. $\rho\left(x=L_{\mathrm{D}}, y=H / 2, t\right)$. The middle panel shows the corresponding wavelet power spectrum with the tadpole pattern (characteristic period $P=6.3 \mathrm{~s}$ ) outlined by contour at the $99 \%$ confidence level. The bottom panel shows the global wavelet spectrum with the maximum for the period $P=6.3 \mathrm{~s}$.

\section{Discussion and conclusions}

For fifteen spikes events we found that their Fourier spectra are power-law spectra with the power-law index in the range of -1.2 
to -1.8 . Moreover, in all these events we recognized the tadpoles in their wavelet spectra, which indicate the propagating magnetoacoustic waves in the spikes sources. When interpreting these spikes as generated in turbulent reconnection outflows we modeled the magnetoacoustic waves and corresponding tadpoles. We found good agreement between the modeled and observed tadpole for the halfwidth of the current sheet $w=1 \mathrm{Mm}$ and for the distance between the location of the perturbation and location of the magnetoacoustic wave detection $L_{\mathrm{P}}-L_{\mathrm{D}}=8.33 \mathrm{Mm}$. For other parameters in the model, see the previous section. Although these parameters are not unique, they give us rough information about the sizes of the global flare current sheet. Especially, the period of the tadpoles has a high diagnostic value. Similarly as for the dense slab (Nakariakov et al. 2004), in our case the period was found to be proportional to the half-width of the current sheet.

In our simulations we consider the Harris current sheet, which covers the whole reconnection region including the reconnection plasma outflows, i.e. the global current sheet. Thus, the halfwidth of the current sheet $(1000 \mathrm{~km})$, estimated from magnetoacoustic waves, means the half-width of this global current sheet (current layer). This width is of the order of the observed current layers, e.g. by Ciaravella et al. (2002) and Lin et al. (2005). We neglected substructures in this global current sheet given by smaller and smaller plasmoids and narrower and narrower current sheets. Spatial scales of this cascade of current sheets come down to a few meters where the current densities are so high that the anomalous resistivity is generated and fast reconnection occurs. An advantage of this model with multiscale current sheets is that it naturally explains the electron fluxes inferred from hard X-ray observations (from all the acceleration sites). For more details about this scale problem, see Bárta et al. (2010a,b).

Because the magnetoacoustic waves are a part of the turbulent reconnection outflow, we consider their finding on the spike sources as supporting our model of spikes. Based on the new findings (Bárta et al. 2010b) and also on observations, we proposed that the narrowband dm-spikes are generated by driven coalescence and fragmentation processes in turbulent reconnection outflows. (Remark: Generally, these processes can be for the whole reconnection region, i.e. also in the reconnection outflow oriented upwards; therefore, the spikes can also be generated at high coronal altitudes as metric spikes.) The propagating magnetoacoustic waves (indicated by tadpoles) modulate these coalescence processes via a modulation of current densities in interaction regions among plasmoids. These waves modulate an acceleration of electrons and generation of plasma and electromagnetic waves - spikes. The narrowband dm-spikes can thus be considered as a radio signature of the fragmented reconnection in solar flares.
Acknowledgements. This research was supported by the grants IAA300030701 (GA AS CR), P209/10/1680 (GA CR), the research project AV0Z10030501 of the Astronomical Institute AS, and the Centre for Theoretical Astrophysics, Prague. Authors thank Dr. J. Rybák for his help with the wavelet analysis that was performed with software based on tools provided by $\mathrm{C}$. Torrence and G. P. Compo at http://paos. colorado. edu/research/wavelets.

\section{References}

Aschwanden, M. J. 1990, A\&AS, 85, 1141

Bárta, M., \& Karlický, M. 2001, A\&A, 379, 1045

Bárta, M., Vršnak, B., \& Karlický, M. 2008a, A\&A, 477, 649

Bárta, M., Karlický, M., \& Žemlička, R. 2008b, Sol. Phys., 253, 173

Bárta, M., Büchner, J., \& Karlický, M. 2010a, Adv. Space Res., 45, 10

Bárta, M., Büchner, J., Karlický, M., \& Skála, J. 2010b, ApJ, submitted [arXiv: 1011.4035B]

Benz, A. O. 1986, Sol. Phys., 104, 99

Benz, A. O., Zlobec, P., \& Jaeggi, M. 1982, A\&A, 109, 305

Chung, T. J. 2002, Computat. Fluid Dyn. (New York, USA: Cambridge University Press)

Ciaravella, A., Raymond, J. C., Li, J., et al. 2002, ApJ, 575, 1116

Fleishman, G. D., \& Yastrebov, S. G. 1994, Sol. Phys., 154, 361

Fleishman, G. D., \& Melnikov, V. F. 1998, Uspechi fizicheskich nauk, 168, 1265 (in Russian)

Fu, Q. J., Li, C. S., \& Yin, S. Z. 1985, in Kunming Workshop on Solar Physics and Interplanetary travelling phenomena, ed. C. de Jager, \& Chen Biao (Beijing: Science Press), 560

Güdel, M. 1990, A\&A, 239, L1

Güdel, M., \& Zlobec, P. 1991, A\&A, 245, 299

Holman, G. D., Eichler, D., \& Kundu, M. 1980, in IAU Symp. 86, ed. M. Kundu, \& T. Gergely, 465

Jelínek, P., \& Karlický, M. 2009, Eur. Phys. J. D, 54, 305

Jelínek, P., \& Karlický, M. 2010, IEEE Trans. Plasma Sci., 38, 2243

Jiřička, K., Karlický, M., Kepka, O., \& Tlamicha, A. 1993, Sol. Phys., 147, 203

Karlický, M. 1984, Sol. Phys., 92, 329

Karlický, M., \& Bárta, M. 2007, A\&A, 464, 735

Karlický, M., Sobotka, M., \& Jiřička, K. 1996, Sol. Phys., 168, 375

Karlický, Jiřička, K., \& Sobotka, M. 2000, Sol. Phys., 195, 165

Karlický, M., Bárta, M., \& Rybák, J. 2010, A\&A, 514, A28

Krucker, S., \& Benz, A. O. 1994, A\&A, 285, 1038

Kuijpers, J., Van der Post, P., \& Slottje, C. 1981, A\&A, 102, 331

Lin, J., Ko, Y.-K., Sui, L., et al. 2005, ApJ, 622, 1251

Magara, T., Mineshige, S., Yokoyama, T., \& Shibata, K. 1996, ApJ, 466, 1054

Melrose, D. B., \& Dulk, G. A. 1982, ApJ, 259, 844

Mészárosová, H., Veronig, A., Zlobec, P., \& Karlický, M. 2003, A\&A, 407, 1115

Mészárosová, H., Karlický, M., Rybák, J., \& Jiřička, K. 2009a, ApJ, 697, L108

Mészárosová, H., Karlický, M., Rybák, J., \& Jiřička, K. 2009b, A\&A, 502, L13

Nakariakov, V. M., Arber, T. D., Ault, C. E., et al. 2004, MNRAS, 349, 705

Priest, E. R. 1982, Sol. Magnetohydrodynamics (London, UK: D. Reidel Publishing Company)

Sankaran, K., Martinelli, L., Jardin, S. C., \& Choueiri, E. Y. 2002, Int. J. Numer. Meth. Engng., 53, 1415

Shibata, K., \& Tanuma, S. 2001, Earth Planets Space, 53, 473

Slottje, C. 1981, Atlas of Fine Structures of Dynamic Spectra of Solar Type IV$\mathrm{dm}$ and Some Type II Radio Bursts, Dwingeloo Observatory

Stähli, M., \& Magun, A. 1986, Sol. Phys., 104, 117

Tajima, T., Benz, A. O., Thaker, M., \& Leboeuf, J. N. 1990, ApJ, 353, 666

Vlahos, L., \& Sharma, R. R. 1984, ApJ, 290, 347

Winglee, R. R., Dulk, G. A., \& Pritchett, P. L. 1988, ApJ, 328, 809

Wentzel, D. G. 1991, ApJ, 373, 285

Zlobec, P., \& Karlický, M. 1998, Sol. Phys., 182, 477 